others were lymphadenopathy, orbital pseudotumor, pancreas and salivary glands in decreasing order (Table). Twenty-four (46.1\%) of patients had localized involvement. Corticosteroids were mainstay of treatment in $92.5 \%$ of patients, and in $57.5 \%$ with any immunosuppressive agents as first line treatment. Rituximab has been used for cases resistant to previous treatment or with relapses in $19(47.5 \%)$ of patients. A complete response was achieved in $52.5 \%$ of patients and partial response ( $<50 \%$ of regression) in $40 \%$. Two patients deceased due to IgG4-RD attributed problems and no malignancy was observed (median follow up: 18 months). Conclusions: We observed similar features with previous European cohorts however no male predominance was seen. Even though conventional immunosuppressives were used in more than half of patients, treatment had switched to rituximab $\sim 50 \%$ patients owing to resistance or relapses.

Disclosure of Interest: None declared

DOI: 10.1136/annrheumdis-2017-eular.4315

\section{FRI0613 H-FERRITIN AND PRO-INFLAMMATORY CYTOKINES ARE INCREASED IN THE BONE MARROW OF ADULT PATIENTS AFFECTED BY MACROPHAGE ACTIVATION SYNDROME}

P. Ruscitti ${ }^{1}$, P. Cipriani ${ }^{1}$, F. Ciccia ${ }^{2}$, P. Di Benedetto ${ }^{1}$, A.R. Lizzi $^{3}$, V. Liakouli ${ }^{1}$, O. Berardicurti ${ }^{1}$, F. Carubbi ${ }^{1}$, G. Guggino ${ }^{2}$, G. D'Andrea ${ }^{3}$, G. Triolo ${ }^{2}$, R. Giacomelli ${ }^{1} .{ }^{1}$ Division of Rheumatology, Department of Biotechnological and Applied Clinical Science, School of Medicine, University of L'Aquila, L'Aquila; ${ }^{2}$ Division of Rheumatology, Department of Internal Medicine, University of Palermo, Palermo; ${ }^{3}$ Department of Biotechnological and Applied Clinical Science, School of Medicine, University of L'Aquila, L'Aquila, Italy

Background: During macrophage activation syndrome (MAS), an inflammatory life-threatening syndrome, extremely high levels of serum ferritin may be observed [1]. Ferritin is an intracellular iron storage protein comprising 24 subunits that may be divided in heavy $(H)$ subunits and light $(L)$ subunits, based on their molecular weight [2]. The $\mathrm{H}$-/L-subunits ratio may change, depending on the specific tissue and the physiologic status of the cell. In the normal condition, ferritin enriched in $L$ subunits ( $L$-ferritin) has been found in the liver and in the spleen, whereas the ferritin enriched in $\mathrm{H}$ subunits ( $\mathrm{H}$-ferritin), may be mainly observed in the heart and kidneys [2]

Objectives: We investigated the tissue expression of both $\mathrm{H}$-and $\mathrm{L}$-ferritin as well as the macrophage subsets expressing these molecules, in the inflammatory BM infiltrate of MAS patients. In addition, the co-expression of IL-1 $\beta, T N F$, IFN- $\gamma$ and $\mathrm{H}$ - or $\mathrm{L}$ ferritin, within the inflammatory cells, was assessed. Finally, we explored if the imbalance between $\mathrm{H}$-ferritin and $\mathrm{L}$-ferritin as well as the number of ferritin positive cells may be considered helpful bio-markers to assess the severity of these patients.

Methods: We analysed the bone marrow (BM) biopsies, by immunofluorescence of 10 adult MAS patients affected by rheumatic disease to assess the presence of: i. both $\mathrm{H}$ - and L-ferritin; ii. the number of CD68+/H-ferritin+ and CD68+/L-ferritin+; iii. the tissue pro-inflammatory cytokines, IL-1 $\beta$, TNF, IFN- $\gamma$; and we correlated these data with clinical and laboratory data. Furthermore, the presence of ferritins was assessed in the sera of the same patients by western blot analysis.

Results: We observed an increased tissue expression of $\mathrm{H}$-ferritin and of proinflammatory cytokines (IL-1 $\beta$, TNF, IFN- $\gamma$ ). Western blot analysis, in the sera, of $\mathrm{H}$-ferritin mirrored data on the tissue. Furthermore, an increased number of CD68+/H-ferritin+ cells and an infiltrate of cells co-expressing $\mathrm{H}$-ferritin and IL-12, suggesting an infiltrate of M1 macrophages, were observed.

Tissue $\mathrm{H}$-ferritin levels correlated with the decreased counts of WBC $(p=0.01)$ and PLT $(p=0.0001)$; with the increased values of serum ferritin $(p=0.012)$ and C-reactive protein (CRP) $(p=0.0058)$; and with the tissue expression of $\mathrm{IL}-1 \beta(p=0.006)$. The number of the $\mathrm{CD} 68+/ \mathrm{H}$-ferritin + cells correlated with the decreased counts of WBC $(p=0.03)$ and PLT $(p=0.0007)$, and with the increased serum ferritin levels $(p=0.0088)$ and CRP $(p=0.049)$. The analyses concerning tissues L-ferritin as well as the number of CD68+/L-ferritin+ cells and the same parameters failed to show any significant result.

Conclusions: We observed an increased tissue expression of $\mathrm{H}$-ferritin associated with an increased expression of IL-1 $\beta$. Interestingly, in the BM inflammatory infiltrate an increased number of $\mathrm{CD} 68+/ \mathrm{H}$-ferritin+ cells was shown. Of note, tissue expression of $\mathrm{H}$-ferritin as well as the number of $\mathrm{CD} 68+/ \mathrm{H}$-ferritin+ significantly were associated with the hematological involvement of the disease, suggesting possible bio-markers to assess the severity of these patients. References:

[1] Ramos-Casals M, et al. Lancet. 2014;383:1503-16.

[2] Rosário C, et al. BMC Med. 2013;11:185.

Disclosure of Interest: None declared

DOI: 10.1136/annrheumdis-2017-eular.5420

\section{FRI0614 FREQUENCY OF ORGAN MANIFESTATIONS IN CHRONIC SARCOIDOSIS}

R. Bergner ${ }^{1}$, K. de Groot ${ }^{2}$, G.A. Müller ${ }^{3}$, P. Korsten ${ }^{3} .{ }^{1}$ Medizinische Klinik A, Klinikum Ludwigshafen, Ludwigshafen: ${ }^{2}$ Medizinische Klinik III, Sana Klinikum Offenbach, Offenbach; ${ }^{3}$ Klinik für Nephrologie und Rheumatologie,

Universitätsmedizin Göttingen, Göttingen, Germany

Background: Chronic sarcoidosis is a systemic disease of unknown etiology, characterized by the histological finding of granulomas in involved organ systems. The most often affected organ is the lung with approximately $90-95 \%$. Systematic data of organ manifestations other than the lung are scarce and show a wide range from $1-2 \%$ up to $50 \%$ depending on the series.

Methods: We analyzed data of newly diagnosed chronic sarcoidosis in 3 tertiary hospitals. We analyzed data on organ manifestations (OM), type of OM and laboratory findings. The certainty of $\mathrm{OM}$ was classified as grade 0 (not investigated), grade 1 (no sign of OM), grade 2 (clinical sign of OM), grade 3 (signs of OM in laboratory findings or imaging) and grade 4 (histological proven $\mathrm{OM})$, respectively.

Results: We included 151 patients with biopsy-proven chronic sarcoidosis. Mean age was $50.8 \pm 15$ years with a male predominance $(87$ [57.2\%] vs. 65 [42.8\%] patients).

Except for 3 patients, all demonstrated pulmonary involvement. The predominant type of lung involvement was type I (mediastinal lymph node enlargement) in $54.2 \%$ and type II (mediastinal lymph node enlargement and interstitial involvement) in $27.7 \%$.

$96.5 \%$ of patients were investigated for an affection of the kidneys, $97.3 \%$ for hepatic, $92.7 \%$ for skin involvement, $68.8 \%$ for ocular manifestations, $67.5 \%$ for ear, nose, throat (ENT) manifestations and $92 \%$ for cardiac manifestations, respectively.

Grade 3 (imaging/laboratory) and grade 4 (histology) findings were seen in the kidneys in $7.6 / 22.8 \%$, in the liver in $13.3 / 11.9 \%$, in the heart in $10.6 / 0.7 \%$, in the eyes in 6.6/

Conclusions: $\mathrm{OM}$ in chronic sarcoidosis are more frequent than suggested in the current literature, especially renal and hepatic. About $20 \%$ of patients with chronic sarcoidosis suffered from moderate to severe CKD due to sarcoidosis, which is a major organ complication contributing to overall morbidity.

We recommend a systematic screening for OM in all patients with chronic sarcoidosis as it is performed in other systemic rheumatic disease.

Disclosure of Interest: None declared

DOI: 10.1136/annrheumdis-2017-eular.6716

\section{FRI0615 SARCOIDOSIS AND CANCER: DIFFERENT PATTERNS OF ASSOCIATION IN A MULTICENTER COHORT FROM SOUTHERN EUROPE}

R. Pérez-Alvarez ${ }^{1}$, B. Kostov ${ }^{2}$, A. González García ${ }^{3}$, R. Gómez De La Torre ${ }^{4}$, M. Lopez Dupla ${ }^{5}$, B. De Escalante ${ }^{6}$, A. Alguacil ${ }^{7}$, J. Chara ${ }^{8}$, J. Velilla ${ }^{9}$, J. Rascón ${ }^{10}$, J.S. Garcia Morillo ${ }^{11}$, C. Tolosa ${ }^{12}$, E. Fonseca ${ }^{13}$, M. Bonet ${ }^{14}$ J.L. Callejass ${ }^{15}$, G. de la Red ${ }^{16}$, E. Calvo ${ }^{17}$, A. Gómez Lozano ${ }^{18}$, E. Peral ${ }^{19}$, J.F. Gómez Cerezo ${ }^{20}$, G. Cruz ${ }^{21}$, P. Perez ${ }^{22}$, S. Rodríguez Fernández ${ }^{23}$, B. Pinilla ${ }^{24}$, A. Gato ${ }^{25}$, M. Akasbi ${ }^{26}$, A. Robles ${ }^{27}$, I. Ojeda ${ }^{28}$, M.J. Vives ${ }^{29}$, C. Morcillo ${ }^{30}$, M. Penadés ${ }^{31}$, M. De Vicente ${ }^{32}$, M. Ramos-Casals ${ }^{33}$, L. Pallarés ${ }^{10}$, P. Brito-Zerón ${ }^{30,33}$ on behalf of the SARCOGEAS-SEMI Registry. ${ }^{1}$ Hosp Alvaro Cunqueiro, Vigo; ${ }^{2}$ IDIBAPS, Barcelona; ${ }^{3}$ Hosp Ramón y Cajal, Madrid; ${ }^{4}$ HUCA, Oviedo; ${ }^{5}$ Hosp Joan XXIII, Tarragona; ${ }^{6}$ Hosp Clínico, Zaragoza; ${ }^{7}$ Hosp Virgen de la Salud, Toledo; ${ }^{8}$ Hosp Josep Trueta, Girona; ${ }^{9}$ Hosp Miguel Servet, Zaragoza; ${ }^{10}$ Hosp Son Espases, Palma de Mallorca; ${ }^{11}$ Hosp Virgen del Rocio, Sevilla; ${ }^{12}$ Hosp Parc Taulí, Sabadell; ${ }^{13}$ Hosp Cabueñes, Gijón; ${ }^{14}$ Althaia,

Manresa; ${ }^{15}$ Hosp San Cecilio, Granada; ${ }^{16}$ Hosp Esperit Sant, Santa Coloma;

${ }^{17}$ Hosp San Jorge, Huesca; ${ }^{18}$ Hosp Sta Caterina, Girona; ${ }^{19}$ Hosp Virgen Macarena, Sevilla; ${ }^{20}$ Hosp Infanta Sofía, Madrid; ${ }^{21}$ Hosp de Poniente, Almería;

${ }^{22}$ Hosp Puerta del Mar, Cádiz; ${ }^{23}$ Hosp da Barbanza, A Coruña; ${ }^{24}$ Hosp Gregorio Marañón, Madrid; ${ }^{25} \mathrm{CH}$, Albacete; ${ }^{26}$ Hosp Infanta Leonor; ${ }^{27}$ Hosp la Paz, Madrid; ${ }^{28}$ Hosp Valle del Guadiato, Córdoba; ${ }^{29}$ San Joan de Déu, San Boi;

${ }^{30}$ Hosp CIMA-Sanitas, Barcelona; ${ }^{31}$ Hosp de Manises, Valencia; ${ }^{32}$ Hosp Nuestra Señora del Prado, Talavera; ${ }^{33}$ Hosp Clínic, Barcelona, Spain

Objectives: To evaluate the temporal association between the diagnosis of neoplasia and sarcoidosis in a large cohort of Spanish patients with sarcoidosis. Methods: In January 2016, the Autoimmune Diseases Study Group (GEASSEMI) created a national registry (SARCOGEAS) of patients with sarcoidosis Sarcoidosis was diagnosed with the criteria proposed by the ATS/ERS/WASOG 1999 statement, and extrathoracic disease by the 2014 WASOG instrument. Diagnosis of neoplasia was recorded before and after the diagnosis of sarcoidosis. Results: The cohort included 1082 patients (82\% biopsy-proven, 618 women, mean age 47yrs). Association with neoplasia was detected in 135 (13\%) patients who developed 140 neoplasms ( 110 solid and 30 hematological neoplasia). The neoplasia more frequently reported were breast $(n=18)$, lymphoma $(n=16)$, non-melanoma skin $(n=15)$ and colon $(n=15)$. Association with neoplasia was more frequent in patients born in Spain $(97 \%$ vs $86 \%, p<0.001$, OR 4.06$)$, older patients ( 55 vs $46 y$ rs, $p<0.001$, OR 1.03 ) and those with bone marrow involvement ( $14 \%$ vs $4 \%, p<0.001$, OR 3.64). Patients in whom cancer preceded the diagnosis of sarcoidosis had a higher frequency of sarcoidosis diagnosed incidentally ( $20 \%$ vs $4 \%, p=0.011)$ and a lower frequency of ocular sarcoidosis ( $3 \%$ vs $16 \%, p=0.016)$. Patients with associated hematological neoplasia had a higher frequency of ENT ( $13 \%$ vs $1 \%, p=0.009)$ and bone marrow $(33 \%$ vs $9 \%, p=0.002)$ involvements in comparison with patients with associated solid neoplasia.

Conclusions: Association between sarcoidosis and cancer was found in $13 \%$ of patients ( $80 \%$ solid and $20 \%$ hematologic malignancies). Elderly patients and those born in Spain were at high risk of having associated cancer. Asymptomatic 
sarcoidosis was more frequently observed in those patients with a previous history of neoplasia, while the association with hematological neoplasms was linked to a higher frequency of sarcoidosis involving ENT and bone marrow.

Disclosure of Interest: None declared

DOI: 10.1136/annrheumdis-2017-eular.3700

\section{FRI0616 EYE MANIFESTATIONS OF PATIENTS WITH MUCKLE-WELLS} SYNDROME

S. Cekic ${ }^{1}$, O. Yalcinbayir ${ }^{2}$, S.S. Kilic ${ }^{1} .{ }^{1}$ Pediatric Rheumatology;

${ }^{2}$ Ophtahlmatology, Uludag University Medical Faculty, Bursa, Turkey

Background: CAPS is a rare autoinflammatory disease associated with mutations in the CIAS1 gene, encoding for NLRP3 that result in overactivation of the inflammasome and systemic inflammation. Muckle-Wells syndrome (MWS) is a rare autosomal dominant disease which causes episodic fever attacks, sensorineural deafness, recurrent hives, arthritis and eye involvement.

Objectives: Here we present the findings of eye involvement in a family whose 11 members have MWS.

Methods: Clinical data was collected during the course of ongoing patient care.

Results: We evaluated the clinical features of 11 patients who were referred to a tertiary care center. The median age of the patients was 25 years (range: 9-65). The ratio of females /males was 1.2 (6/5). All patients had arthritis with exacerbation on exposure to cold and recurrent episodes of pink eye. The median age of onset of ocular involvement was 8 years (2-45). We observed severe eye involvement in $36 \%$ of our cases, including band keratopathy, severe damage of corneal stroma and neovascularization. Corneal involvement and clouding was detected in four patient. Two of those had the diagnosis of keratoconus as well. Patients with keratoconus had corneal scarring due to corneal hydrops verified with corneal topography. The other two patients with corneal clouding had bant keratopathy. One of those patient was a 17 year old girl who had recurrent uveitis with hypopyon which necessiated the use of intravitreal dexamethasone implant. She also had posterior synechia of the iris to the lens. The other eye of that patient had signs of phthisis bulbi. The other patient with bant keraopathy was 46 years old male who had optic atrophy and tractional fibrovascular membranes at the posterior pole of the eye. Anakinra was used for treatment of 5 cases, and canakinumab of 3 cases. It was observed that the frequency of conjunctivitis decreased after anti IL-1 therapy. There was no mutation detected in the study of MEFV (all exons), TNFRSF1A (exons 2 to 7), MVK (all exons), NLRP3 (all exons), NOD2 (exons 4, 8 and 9) and PSTPIP1 (exons 10 and 11) genes.

Conclusions: In this study, it has been shown that eye findings related to MWS can vary from conjunctivitis to severe uveitis. We want to emphasize that ocular involvement in MWS should be carefully assessed, since it can lead to visual impairment.

Disclosure of Interest: None declared

DOI: 10.1136/annrheumdis-2017-eular.3352

\section{FRI0617 DIAGNOSTIC SENSITIVITY OF CUTOFF VALUES OF IGG4-POSITIVE PLASMA CELL NUMBER AND IGG4-POSITIVE/CD138-POSITIVE CELL RATIO IN TYPICAL MULTIPLE LESIONS OF PATIENTS WITH IGG4-RELATED DISEASE}

S. Tsuge, I. Mizushima, Y. Fujisawa, S. Hara, F. Suzuki, K. Ito, H. Fujii, K. Yamada, M. Kawano. Division of Rheumatology, Department of Cardiovascular and Internal Medicine, Kanazawa University hospital, Kanazawa, Kanazawa, Japan

Background: Immunoglobulin G4-related disease (IgG4-RD) is a recently recognized systemic inflammatory disease with multi-organ involvement [1]. Diagnostically, two of the most important hallmarks of IgG4-RD are high IgG4-positive plasma cell (PC) counts and high IgG4/lgG ratios in affected organs. Although the International consensus statement (ICS) on the pathology of IgG4-RD adopted different IgG4-positive PC counts among affected organs for the diagnosis to differentiate IgG4-RD mimickers from IgG4-RD [2], histological and immunohistochemical findings of the specimens from not only one but multiple organs in the same patient has not been evaluated.

Objectives: This study aimed to investigate the diagnostic sensitivity of the cutoff values of IgG4-positive PC number and lgG4-positive/CD138-positive cell ratio proposed by the International consensus statement (ICS) on the pathology of IgG4-RD in typical multiple lesions of patients with IgG4-RD.

Methods: We evaluated IgG4-positive PC number and IgG4-positive/CD138positive cell ratio in 35 samples from 16 IgG4-RD patients having more than two typical lesions of IgG4-RD.

Results: We evaluated twelve submandibular, eleven ophthalmic, four skin, four kidney, two pancreatic, and one bronchus and prostate lesion each in 16 patients with typical clinical, serological, and radiographic features. Concerning IgG4+ PC number per high power field, most ophthalmic (8/11), kidney (4/4), pancreatic $(2 / 2)$, and bronchial lesions (1/1) fulfilled the cutoff value of ICS, whereas many of the submandibular (5/12) and skin lesions (0/4) did not. In contrast to the absolute number, almost all lesions fulfilled the cutoff value of IgG4+/CD138+ cell ratio. In five cases, only one or two lesions in the same patient fulfilled the cutoff value of ICS, while the others did not.
Conclusions: These results suggest that ICS criteria have different sensitivities among the affected organs in diagnosing IgG4-RD.

References:

[1] Stone JH, et al. IgG4-related disease. N Engl J Med. 2012;366:539-51.

[2] Deshpande V, et al. Consensus statement on the pathology of IgG4-related disease. Mod Pathol. 2012;25:1181-92.

Disclosure of Interest: None declared

DOI: 10.1136/annrheumdis-2017-eular.4170

\section{FRI0618 ADALIMUMAB IN NON-INFECTIOUS UVEITIS - EFFICACY ACROSS DIFFERENT ETIOLOGIES IN THE VISUAL I AND VISUAL II TRIALS}

P.T. Merrill ${ }^{1}$, A. Vitale ${ }^{2}$, M. Zierhut ${ }^{3}$, E. Forton ${ }^{4}$, H. Goto ${ }^{5}$, M. Kron ${ }^{6}$, S. Tari ${ }^{7}$, S. Pathai ${ }^{8} \cdot{ }^{1}$ Rush University Medical Center, Chicago; ${ }^{2}$ University of Utah, Salt Lake City, United States: ${ }^{3}$ University of Tuebingen, Center of Ophthalmology, Tuebingen, Germany; ${ }^{4}$ University of Montreal, Montreal, Canada; ${ }^{5}$ Tokyo Medical University, Tokyo, Japan; ${ }^{6}$ AbbVie Deutschland GmbH \& Co KG, Ludwigshafen, Germany; ${ }^{7}$ AbbVie Inc., North Chicago, United States; ${ }^{8}$ AbbVie Ltd, Maidenhead, United Kingdom

Background: There is increasing interest in understanding the efficacy of adalimumab across different etiologies of uveitis. No prospective analysis has been conducted to date to determine the efficacy of adalimumab among non-infectious uveitis patients with different etiologies.

Objectives: To assess adalimumab (ADA) efficacy in active and inactive, noninfectious uveitis across different etiologies in patients who were recruited as part of the VISUAL program.

Methods: Exploratory data analyses from two global phase 3, double-masked trials: VISUAL I (patients with active uveitis despite $>2$ weeks of prednisone 10-60 mg/day) and VISUAL II (patients with inactive disease dependent on 10-35 $\mathrm{mg} /$ day of prednisone to maintain inactivity) were performed. Patients received placebo (PBO) or ADA subcutaneously ( $80 \mathrm{mg}$ week 0 , followed by $40 \mathrm{mg}$ every other week from week 1 up to 80 weeks). In VISUAL I, all patients received a prednisone burst followed by taper to $0 \mathrm{mg}$ by week 15 . In VISUAL II, prednisone taper to $0 \mathrm{mg}$ was mandatory by week 19 . The primary endpoint was time to treatment failure (TF) at or after week 6 for VISUAL I; and at or after week 2 for VISUAL II ${ }^{1,2}$. For this analysis, patients were categorized into different uveitis etiologies which they presented at study entry. Hazard ratios (HR) for time to TF were obtained for each uveitis etiology.

Results: The efficacy of ADA was significantly greater than PBO in the largest subgroup of patients with Idiopathic/other uveitis (VISUAL I: 103 and VISUAL II: 90) etiology in both VISUAL I' and VISUAL II trials. All other subgroups showed

Figure: Hazard ratios of time to treatment failure by uveitis etiologies. VISUALI (A) and VISUAL I* (B) clinical trials.

A. VISUALI

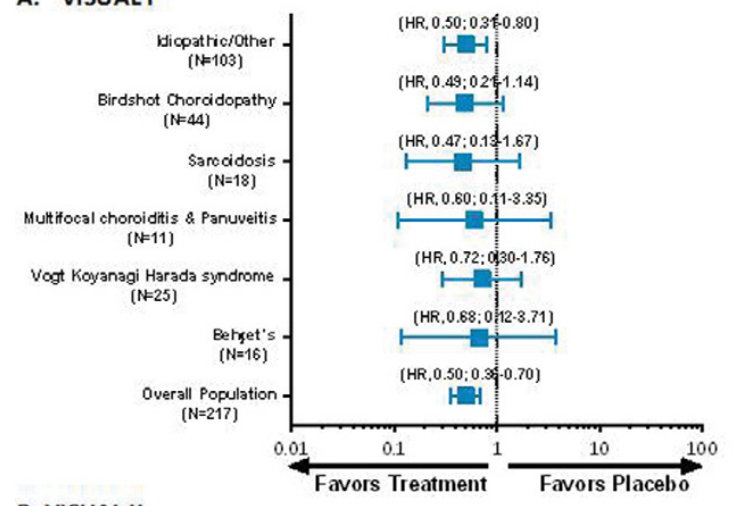

B. VISUAL II

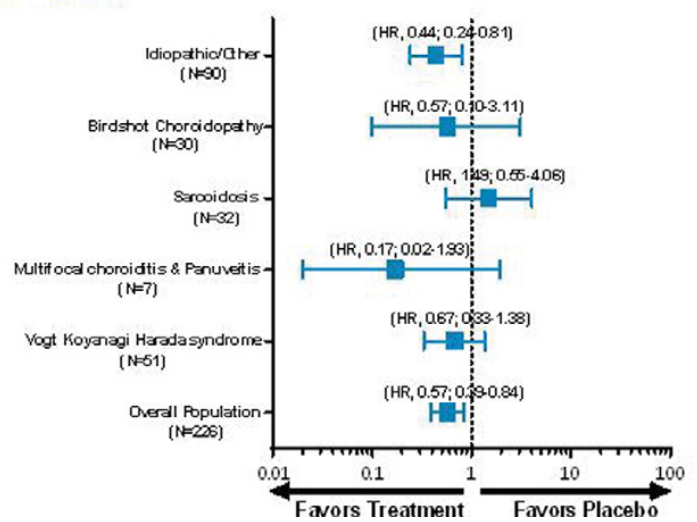

